\title{
Coherent Electron Interference of Diffracted Beams from Amorphous Materials
}

\author{
Rodney A. Herring \\ CAMTEC, MENG, University of Victoria, British Columbia V8W 2Y2 Canada
}

Two interference methods producing high contrast fringes from the diffraction intensities of amorphous materials have been made possible [1, 2]. Recent methods of k-space interference by means of an electron biprism have revealed that all electron intensities on the diffraction plane have sufficient coherence to form fringes. The intensities from amorphous materials are of interest because they represent their atomic structure, which to date has not been solved because of their missing phase information [3]. Relative phase information of the amorphous structure is possible using a wavefront-splitting method [1], whereas, absolute phase information of the amorphous structure, the truly desired information necessary for determining its structure factor, is obtainable using an amplitude spitting method (Fig. 1) [2]. In this method an amorphous layer exists on the surface of a crystal. The crystal's Bragg diffracted beams are used to carry the amplitudes and phases of the amorphous thin film. Interferograms were produced from the diffuse, speckle and ring intensities of many amorphous materials including a-C, a-Si, a-Ge, $\mathrm{SiO}_{2}$ , a-W, a-GaAs and an amorphous metal. The fringes in the interferograms were produced from electrons scattering from low to high angles further than the third amorphous ring in some cases. The absolute phase can then be related to the amorphous intensity's radial distribution for determination of their structure factors. The spatial frequency of the fringes carrying the phase information of the amorphous specimen, which in many cases determines the phase resolution limit, depends on the angle of overlay of the interfering Bragg diffracted beams with the higher spatial frequency Bragg beams transferring more specimen information in the interferogram. If the two crystal beams have equal but opposite phases, i.e., $\pm g_{h k l}$, they cancel when interfered, leaving only two times the absolute amorphous phase,

$$
\Delta \phi=\phi_{g_{h k l}}+\phi_{a m o r}+\phi_{g_{\bar{h} \bar{k}}}+\phi_{\text {amor }}=2 \phi_{\text {amor }} \text {. }
$$

A fly in the ointment for determining the absolute phase of the amorphous specimen is that for convergent beams, there are still geometric unknowns such as the cross-over or focus probe position within the specimen giving rise to uncertainties in the part of the specimen that the left and right sides of the beam pass (Fig. 2). This shortfall is corrected by using a planar beam. First the planar beam passes through the amorphous layer and then it passes through the thin crystal producing the two carrier beams that are interfered as schematically shown in Fig 3. This method of beam interference offers the promise of being able to determine the phase of short- and longrange structures of amorphous materials.

1. Herring R A, Saitoh K, N, Tanaka N, and Tanji T (2010) Coherent Electron Interference from Amorphous TEM Specimens. J. Electron Microscopy 59: 321.

2. Herring R A, Saitoh K, N, Tanji T, and Tanaka N (2012) Electron interference from an amorphous thin film on a crystal transmission electron microscopy specimen. J. Electron Microscopy 61: 17.

3. Howie A (2004) Progress in Determining the Structure of Amorphous and Disordered Materials. Microsc Microanal 10 (Suppl 2) 784.

Grants from JSPS, UVic, NSERC, CFI and BCKDF are gratefully appreciated. 


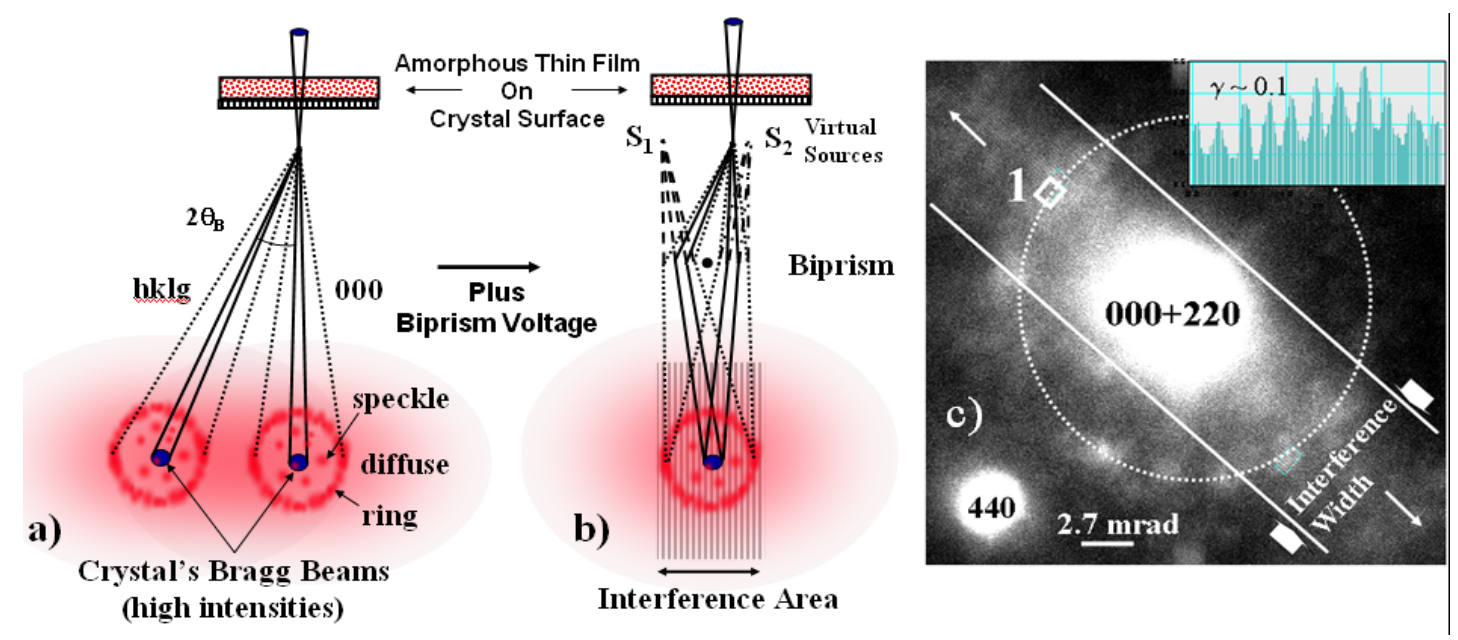

Fig. 1 - DBI of amorphous intensities showing in a) the amorphous intensities being carried by the Bragg diffracted beams, b) the interference of the amorphous intensities using a biprism, c) interference of an amorphous layer on a GaAs crystal. Fringes in box 1 shown in insert.

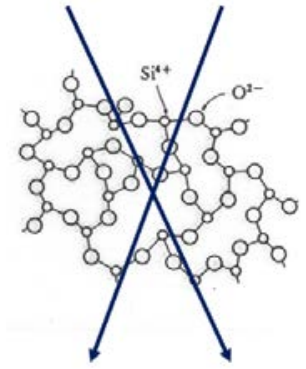

Convergent Beam

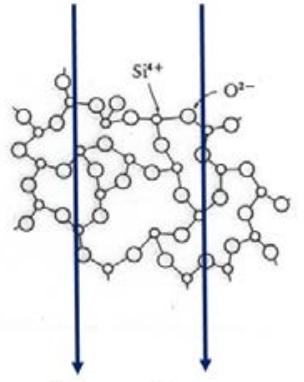

Planar Beam

Fig. 2 - Amorphous structure information difference between convergent and planar beam configurations.

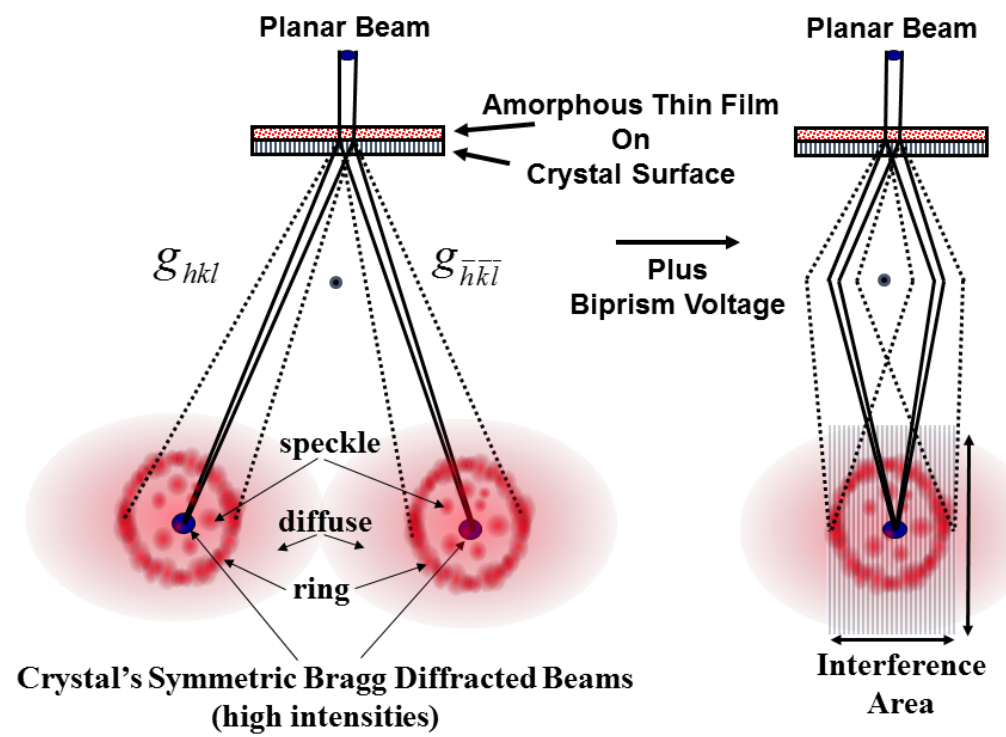

Fig. 3 - Interference of two planar, symmetrically Bragg diffracted beams from a crystal carrying amorphous amplitude and phase information. Interference is by means of an electron biprism. 